\title{
Numerical characterization of Symmetric Delamination in Orthotropic plate using Lamb waves
}

\author{
Salah Nissabouri ${ }^{1}$, Mhammed El Allami ${ }^{2}$, El hassan Boutyour ${ }^{3}$ \\ \{s.nissabouri@uhp.ac.ma ${ }^{1}$,m.elallami@gmail.com ${ }^{2}$,Boutyour.elhassan@gmail.com ${ }^{3}$ \} \\ Labo LMEET, Department of physics applied, FST-Settat, Morocco ${ }^{1,3}$, Labo LMEET, Department of \\ applied physics, FST-Settat, CRMEF-Settat, Morocco $^{2}$
}

\begin{abstract}
In this paper we study numerical modeling of Lamb waves propagation and its interaction with the edges of symmetric delamination in unidirectional orthotropic plate $[0]_{4}$. The simulations were carried out using ABAQUS CAE by exciting the fundamental $\mathrm{A}_{0}$ Lamb mode in the frequency $300 \mathrm{kHz}$. The delamination was then characterized by analyzing the signal picked up at two sensors using two technics: two Dimensional Fast Fourier Transform (2D-FFT) to identify the propagating and converted modes, and Wavelet Transform (WT) to measure the arrival times. The results showed that the mode $\mathrm{A}_{0}$ is sensible to symmetric delamination. Besides, based on signal changes with the delamination edges, a localization method is proposed to estimate the position and the length of the delamination.
\end{abstract}

Keywords: Delamination, orthotropic, Lamb waves, converted modes.

\section{Introduction}

Composites have been widely used to improve mechanical proprieties of structures. They are used in many fields especially in aeronautic industry [1]. Their characteristics are influenced by the proportions of the matrix and the reinforcements. There are other parameters that also affect the properties of a composite like: size, orientation and distribution of the fibers. However, the drawback of composites is their heterogeneity which leads to their weakness and facilitates the appearance of internal and external damages such as: fiber breakage, matrix cracking, through-thickness hole, local delamination. Among these damages, delamination is especially easy to appear, it causes wave scattering, mode conversion, and multiple reflections. To understand these mechanisms, theoretical, numerical and experimental studies are conducted.

Bo Feng, Artur Lopes Ribeiro, Helena Geirinhas Ramos [2] analyzed the interaction of fundamental symmetric $\left(\mathrm{S}_{0}\right)$ and anti-symmetric $\left(\mathrm{A}_{0}\right)$ mode Lamb waves with the delamination using finite element simulations. Guo, Cawley [3] studied by finite element analysis and by experiment the interaction of the $S_{0}$ Lamb mode with delamination. $\mathrm{Ng}$ [4] presented a theoretical and Finite Element $(\mathrm{FE})$ investigation of the scattering characteristics of the fundamental anti-symmetric $\left(\mathrm{A}_{0}\right)$ Lamb wave at delamination in a quasi-isotropic composite laminate. $\mathrm{Ng}$, Veidt [5] studied the influence of stacking sequence on scattering characteristics of the fundamental anti-symmetric Lamb wave at through holes in composite laminates. Yang [6] investigated some aspects of numerical simulation of excitation and detection of Lamb waves using piezoelectric disks in plate like composite laminates. 
In a previous paper [7] we established the theoretical model of lamb waves' propagation in orthotropic plate. To get deeper insight of these phenomena, this work proposes a numerical study to simulate the lamb waves' interaction with symmetrical delamination

\section{Numerical model using Finite Element Method}

Many researchers have used the numerical simulation to study lamb waves. The finite element method has been extensively and successfully used to model the Lamb waves and study their interactions with defects in structures [1] [2] [8].

Chiua , Roseb, Nadarajaha [9] built a 3D continuum model in ABAQUS of the 8 ply laminate with a symmetric stacking sequence $[45 /-45 / 0 / 90]_{S}$. Ng, Veidt [10] used a threedimensional Finite Element (FE) method to simulate an eight-ply [45/-45/0/90]S quasiisotropic composite laminate with a delamination. The simulations were carried out by ANSYS. Panda [11] presented 3D FE simulations that were carried out to visualize the wave propagation and their interaction with the defect at various depths of an 8-layered Glass Fiber Reinforced Polymer (GFRP) composite laminate using the commercial finite element software package ABAQUS/Explicit. Ramadas [12] investigated the interaction of the primary antisymmetric mode, $\mathrm{A}_{0}$ with symmetric delamination type defects in a quasi-isotropic laminated composite using 2D model ANSYS.

In this paper we try to predict the interaction of $\mathrm{A}_{0}$ mode in orthotropic plate. The $2 \mathrm{D}$ numerical simulations were carried out using ABAQUS CAE. The plate has a length $\mathrm{L}=400$ $\mathrm{mm}$ (in the direction of propagation $\mathrm{X}_{1}$ ) and a thickness $\mathrm{h}=1.6 \mathrm{~mm}$. The mechanical properties of each lamina are shown in Table 1. Once the geometry of the plate has been achieved, it remains to mesh and define a sampling sufficient time.

Table 1. Elastic coefficients $\mathrm{C}_{\mathrm{ij}}(\mathrm{Gpa})$ and density $\left(\mathrm{kg} / \mathrm{m}^{3}\right)$ of the materiel

\begin{tabular}{ll}
\hline \multicolumn{1}{c}{$\rho$} & 1530 \\
\hline $\mathrm{C}_{11}$ & 56.9 \\
$\mathrm{C}_{22}=\mathrm{C}_{33}$ & 14.7 \\
$\mathrm{C}_{12}=\mathrm{C}_{13}=\mathrm{C}_{23}$ & 9.76 \\
$\mathrm{C}_{55}=\mathrm{C}_{66}$ & 4.16 \\
\hline
\end{tabular}

\subsection{Model without defect}

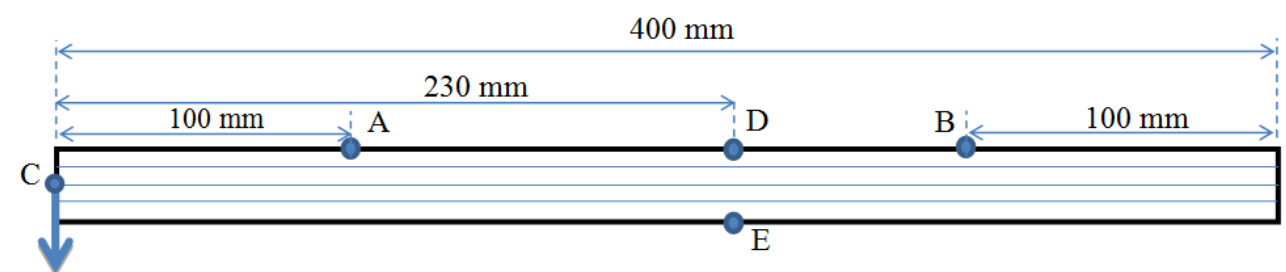

Fig. 1. Numerical Model without defect, C: actuator, A, B, D, E: sensors. 
The out of plan displacements measured at the sensors are sinusoidal with the same amplitude. To verify the excitability of pure mode $\mathrm{A}_{0}, 2 \mathrm{D}-\mathrm{FFT}$ was performed at the sensor A. A matrix was calculated at uniformly spaced points.

\section{D- FFT analysis}

Propagating Lamb waves are sinusoidal in both the frequency and spatial domains. Therefore, a temporal Fourier transform may be carried out to go from the time to frequency domain, and then a spatial Fourier transform may be carried out to go to the frequency wavenumber domain, where the amplitudes and wave Numbers of individual modes may be measured. Applying spatial Fourier methods in practice to data gained experimentally or numerically requires us to carry out a two-dimensional Fourier transform giving:

$$
H(k, f)=\iint_{-\infty}^{+\infty} u(x, t) e^{-i(k x+\omega t)} d x d t
$$

Where $\mathrm{k}$ is the wave number, $\mathrm{f}$ is the frequency, $\omega=2 \pi \mathrm{f}$. The result of this transformation will be a two-dimensional array of amplitudes at discrete frequencies and wavenumber. [13]



Fig .2. Superposition of FFT2D over analytical dispersion curves

The Fig. 2 shows that there is only one mode propagating which is $\mathrm{A}_{0}$.

\subsection{Model with symmetric delamination}



Fig .3. Snapshot of FE simulated out-of plane displacement in the upper and the lower sub-laminates separated by the delamination. 
The Fig.3 is a snapshot of FE out-of plane displacement. The Lamb waves split into two parts and travel independently with different velocities in the upper and lower sub-laminates. Mode conversions happen at the two edges of a delamination due to the change of boundary conditions. To characterize the delamination, we analyzed the signal picked up at the sensors $\mathrm{A}$ and $\mathrm{B}$.

\section{Results}

\subsection{Signal picked up at Sensor A}

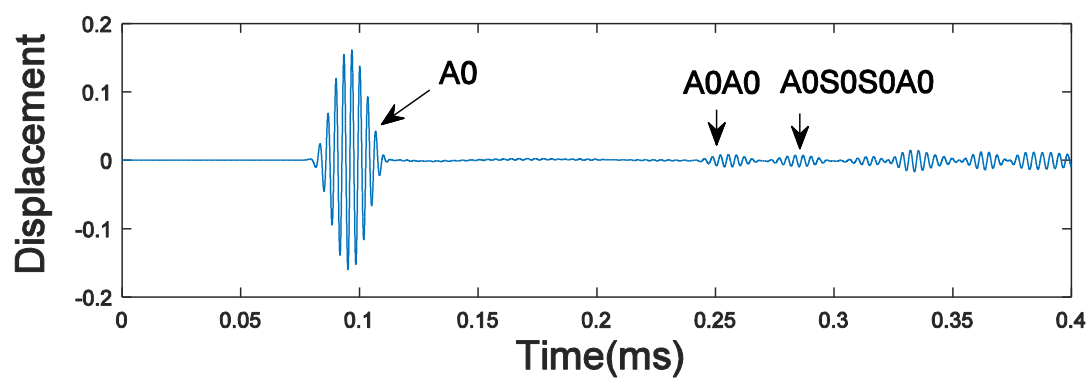

Fig. 4. Out-of-plane displacement, taken on damaged composite $[0]_{4}$ at $\mathrm{x}=100 \mathrm{~mm}$, case delamination length 60 between lamina 1 and lamina 2 .

The Fig. 4 is A-scan image from the numerical simulations, taken at $100 \mathrm{~mm}$ for a delamination length of $60 \mathrm{~mm}$. It showed also the incident wave $\mathrm{A}_{0}$ and the two reflected waves: $\mathrm{A}_{0} \mathrm{~A}_{0}, \mathrm{~A}_{0} \mathrm{~S}_{0} \mathrm{~S}_{0} \mathrm{~A}_{0}$.

\subsection{Delamination Characterization from data acquired at sensor A}

Wavelet transform is an important tool in the time frequency domain of transient signals. This information is independent of where in time this component appears. The continuous wavelet transform of signal $\mathrm{u}(\mathrm{t})$ is defined as follows:

$$
\operatorname{cwt}(a, b)=\frac{1}{\sqrt{|a|}} \int_{-\infty}^{+\infty} u(t) \psi^{*}\left(\frac{t-b}{a}\right) d t
$$

The transformed signal is a function of two variables, a and $b$, the translation and scale parameters, respectively. Psi(t) is the transforming function, and it is called the mother wavelet. In this paper we use wavelet transform by 'Gauss 1' to locate the pic which permits to determine the arrival time of the wave at specific frequency. 


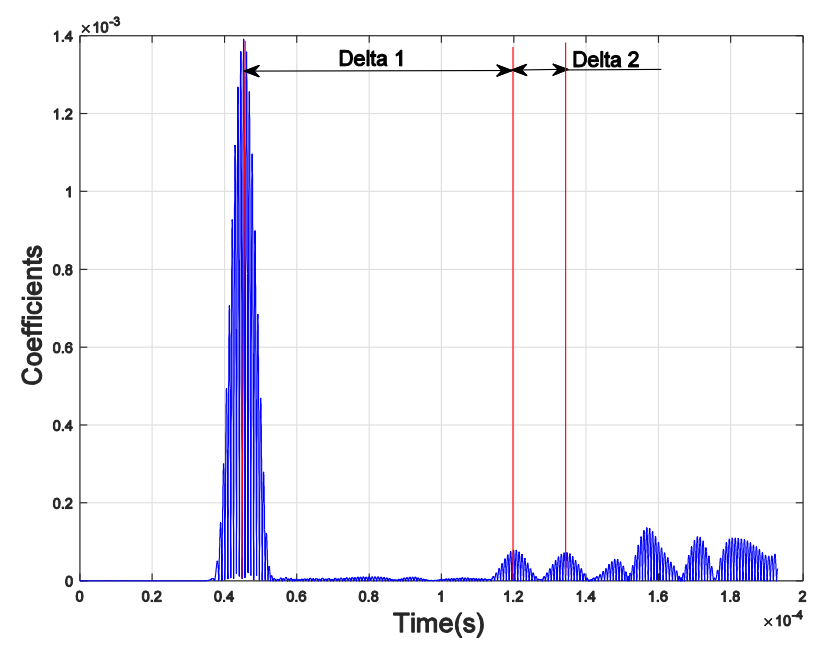

Fig. 5. Coefficients of wavelet gauss1, delamination length 60

The Fig. 5 presents wavelet coefficients versus time at sensor A. The time delay between the peaks permits to estimate the position and the length of delamination. Knowing the group velocity of the modes $A_{0}$ and $S_{0}: \operatorname{Vg}\left(A_{0}, 300 \mathrm{khz}\right)=1268 \mathrm{~m} / \mathrm{s}, \operatorname{Vg}\left(S_{0}, 300 \mathrm{khz}\right)=5649 \mathrm{~m} / \mathrm{s}$, we can calculate the delamination position from the sensor $A$ noted $P_{D}$ by $\Delta t_{1}$, and delamination length noted $\mathrm{L}_{\mathrm{D}}$ by $\Delta \mathrm{t}_{2}$ as presented in table below :

Table 2. Delamination charactrization : length and position (mm)

\begin{tabular}{cccccc}
\hline Length & $\mathrm{P}_{\mathrm{D}}$ & $\mathrm{P}_{\mathrm{DTH}}$ & Error \% & $\mathrm{L}_{\mathrm{D}}$ & Error \% \\
\hline 60 & 103,2 & 100 & 3,2 & 62,2 & 3,7 \\
90 & 106,6 & 100 & 6,6 & 94,1 & 4,5 \\
120 & 103,1 & 100 & 3,1 & 120,9 & 0,8 \\
\hline
\end{tabular}

The sensor A permits to evaluate the position and the length of symmetric delamination. The Errors associated with the predicted symmetric delamination position range from $3,1 \%$ to $6,6 \%$. As far as the length prediction is concerned, the errors range from $0,8 \%$ to $3,7 \%$. So we conclude that more the delamination is long more the length estimation is high.

\subsection{Signal picked up at Sensor B}

The Fig. 6 represents different wave modes in the case of $\mathrm{A}_{0}$ mode is incident on the transmission side of the main laminate at sensor $B$. A new mode $S_{0}$ was generated when the $A_{0}$ interacted with the entrance of delamination as showed in the figure 6 . This new mode traveled within the upper sub-laminates along the length of delamination. Then it reached the exit of delamination, interacted with the exit and generated a new mode $\mathrm{A}_{0}$ in the forward direction. 




Fig. 6. Out-of-plane displacement, taken on damaged composite $[0]_{4}$ at $\mathrm{x}=300 \mathrm{~mm}$. case delamination length 60

Table 3. Delamination charactrization : length (mm)

\begin{tabular}{ccc}
\hline Length & $\mathrm{L}_{\mathrm{D}}$ & Error \% \\
\hline 60 & 62,9 & 4,9 \\
90 & 94,0 & 4,5 \\
120 & 122,5 & 2,1 \\
\hline
\end{tabular}

The sensor B permits to evaluate only the length of symmetric delamination. The Errors associated with the predicted symmetric delamination length range from $2,1 \%$ to $4,9 \%$.

\section{Conclusion}

In this work, we have studied the numerical model describing the propagation of antisymmetric mode $\mathrm{A}_{0}$ within an orthotropic plate. Simulations based on Finite Element Method were carried out using ABAQUS Software in order to investigate the interaction of Lamb wave mode by symmetric through thickness delamination. The results showed the conversion phenomenon. To quantify the conversion and to characterize the delamination, we have computed the 2D FFT in order to identify the propagating and converted modes; after that, the WT was calculated at different sensors to measure the arrival times. Analyzing the signal obtained at sensors $\mathrm{A}$ and $\mathrm{B}$, it was found that the mode conversions happen at the edges of a delamination for $\mathrm{A}_{0}$ wave. In fact, this mode is sensitive to the position and to the length of delamination; this makes it become an effective tool to evaluate the delamination in orthotropic materiel. Besides, based on signal changes with the delamination edges, a localization method is proposed to estimate the position and the length of the delamination. These are the main results:

- The estimation is accurate when the delamination length increases.

- The optimal position to evaluate the beginning and the length of delamination is between the actuator and the defect: the pulse echo method.

\section{References}

[1] Bo Feng, Artur Lopes Ribeiro, Helena Geirinhas Ramos, Interaction of Lamb waves with the edges of a delamination in CFRP composites and a reference-free localization method for delamination, Measurement ,doi: https://doi.org/10.1016/j.measurement.2017.10.016. (2017) 
[2] N. Guo a and P. Cawley,The interaction of Lamb waves with delaminations in composite laminates. The Journal of the Acoustical Society of America 94, 2240,(1993)

[3] Ching-Tai Ng, Analytical and finite element prediction of Lamb wave scattering at delaminations in quasi-isotropic composite laminates. Journal of Sound and Vibration, 4870-4883, (2012)

[4] Martin Veidt and Ching-Tai Ng. Influence of stacking sequence on scattering characteristics of the fundamental anti-symmetric Lamb wave at through holes in composite laminates. J. Acoust, 129, (2011)

[5] A De Luca, Numerical simulation of the Lamb wave propagation in impacted CFRP laminate, Procedia Engineering, 109 - 115, (2016)

[6] Chunhui Yang, Some aspects of numerical simulation for Lamb wave propagation in composite laminates, Composite Structures, 267-275,( 2006)

[7] S. Nissabouri, M. El Allami, E. Boutyour, A. Errkik, Lamb wave interaction with delamination in orthotropic plate, International Conference on Computing and Wireless Communication Systems, , Laarche, Maroc, ISBN: 978-1-4503-5306-9 , doi>10.1145/3167486.3167545, (2017)

[8] R. Basri and W.K. Chiu, "Numerical analysis on the interaction of guided Lamb waves with a local elastic stiffness reduction in quasi-isotropic composite plate structures", Composite Structures 66 (2004) 87-99.

[9] W.K. Chiua , L.R.F. Roseb and N. Nadarajaha, "Scattering of the fundamental anti -symmetric Lamb wave by a midplane edge delamination in a fiber-composite laminate". Procedia Engineering $188,317-324,2017$.

[10] Ching-Tai Ng and Martin Veidta, "Scattering of the fundamental antisymmetric Lamb wave at delaminations in composite laminates". Acoustical Society of America 132, 115, 2012.

[11] Rabi Sankar Panda, "Lamb wave interactions with delaminations in composite laminates using aircoupled ultrasonic visualization". 14th Asia-Pacific Conference on Non-Destructive Testing, 2013.

[12]C Ramadas. "Interaction of the primary anti-symmetric Lamb mode (A0) with symmetric delaminations: numerical and experimental studies". Smart Materials and Structures, 2009.

[13] D. Alleyne and P. Cawley, "A two-dimensional Fourier transform method for the measurementof propagating multimode signals”, J. Acoust.Soc. Am. 89 (3), March 1991. 